\title{
Brushed under the carpet: Examining the complexities of participatory research
}

\author{
Caroline Lenette* - University of New South Wales, Australia \\ Nelli Stavropoulou - Durham University, UK \\ Caitlin Nunn - Manchester Metropolitan University, UK \\ Sui Ting Kong - Durham University, UK \\ Tina Cook - Liverpool Hope University, UK \\ Kate Coddington - University at Albany, State University of New York, USA \\ Sarah Banks - Durham University, UK
}

\begin{abstract}
Participatory research is sometimes difficult and risky, but there is a paucity of opportunities - and some reluctance - to reflect on its challenging aspects. In this article, we present subjective accounts of our everyday experiences of conducting participatory research as women researchers. We focus on four themes from our combined research experiences to explore some of the frustrations we encounter in participatory research. We argue that it is crucial to identify, reflect upon and address such aspects in academic outputs to broaden debates and scholarly discussions. We offer these reflections, and related strategies, as a contribution to critical debates on participatory research practice.
\end{abstract}

Keywords: gendered fieldwork, co-research, reflexivity, negotiating roles, practice frameworks, power differentials

\section{Key messages}

- There are challenging aspects to participatory research that are seldom addressed in academic publications, making it difficult to reflect on and learn from risky and difficult research experiences.

- This paper synthesizes a number of field-related issues from subjective perspectives to contribute to critical discussions on participatory research practice and support new and established researchers.

- We share our subjective experiences on the risky and difficult aspects of participatory research as a provocation to others across disciplines and geographical locations to similarly share their challenges and strategies.

\section{Introduction}

Participatory research is a process whereby people with lived experiences of the topic of study are co-creators of knowledge (Abma et al., 2019; Cook et al., 2017; Lenette, 2017; Nunn, 2017). It involves people with direct experiences of, or interest in, the topic of study in all or some aspects of the research process, including research design, data 
collection, analysing findings, and reporting and dissemination. Participatory research begins from a social, ethical and moral commitment not to treat people as objects of research but, rather, to recognize and value the differing and diverse experiences and knowledge of all those involved (see, for instance, Southby, 2017). We subscribe to the view that, irrespective of discipline, participatory research can be used to challenge one or several sociopolitical inequities (Mayan and Daum, 2016). This article emerges from collaborative discussions among a group of women researchers on the lack of opportunities to reflect on the gendered, and sometimes risky, aspects of participatory research. As a group of researchers who use participatory research approaches, we find that we are often reluctant to openly discuss difficulties associated with participatory research for fear of discrediting the approach. In other words, discussing the challenges presented by participatory research can be perceived to create suspicion about the quality of knowledge produced. While participatory research is a useful and sometimes empowering approach, we know that not all experiences or elements of participatory research are positive. As Foster (2016: 68) comments, in practice, implementing participatory research models takes 'a great deal of time and energy to work through, and extensive emotional labour in terms of forging and maintaining meaningful relationships. This is not always recognised in research accounts.' As such, this important topic warrants a reflexive writing process that attends to the more demanding aspects of participatory research from the perspectives of women academic researchers.

In this article, we explore participatory research collaborations between university-based researchers and non-academic community-based researchers, to 'cocreate' knowledge. While there are clear benefits to participatory research, such as the creation of a meaningful research space where genuine collaborative research is possible (Lenette et al., 2013; Nunn, 2017) - and despite our best efforts to ensure that risks are minimized in the field - unforeseen events can lead to increased risks or ethical dilemmas, both for community-based and academic researchers.

The relative paucity of discussions of sensitive topics linked to the methodology can inhibit debate about key challenges in participatory research. In this paper, we critically reflect on incidents, anxieties, decisions and dilemmas that most of us either intentionally repressed or had not dared to mention in our publications until we came together as a group to share our experiences. These issues are not new per se (see, for instance, Banks et al., 2013; Durham Community Research Team, 2011; Southby, 2017; Banks and Brydon-Miller, 2019; Cook, 2012), but the limited literature and reflexive discussions on these difficult topics suggest that we might productively contribute gender-specific perspectives on such issues. As we collectively reflected on our research experiences, it was impossible to ignore the gendered aspect of these issues. As women researchers, we are called upon, or expected, to conduct 'emotional labour', and so from our perspective, participatory research has a distinct gendered dimension.

We draw on our combined research knowledge to discuss institutional, intellectual and relational risks openly, using our subjective experiences of participatory research to highlight aspects that are usually 'brushed under the carpet' - perhaps as a result of institutional pressures or self-censorship - and seldom addressed in academic publications (including our own). Having a space to candidly reflect on these difficult elements is an exercise in reflexivity, which is integral to ethical research practice (Guillemin and Gillam, 2004), and particularly relevant for proponents of participatory research. The purpose of this paper is not to discourage the use of participatory research. In fact, our discussions have reinforced our commitment to such approaches, 
and affirmed the benefits for knowledge production and social justice when projects are conducted in ethical, respectful and mutually beneficial ways. Rather, we want to acknowledge the 'joys and risks of balancing together on a trampoline' (Liamputtong and Rumbold, 2008: 10), which is what some participatory research collaborations feel like. We echo Wright et al.'s (2012: 44) observation that 'the realities of our work together [that is, collaborative work] are much more complex; sometimes they are fragile or confusing, and often they involve unexpected turns'. We seek to present subjective accounts of our everyday, real-life participatory research work that focus specifically on the particularities of conducting participatory research that builds on reflective work on this approach. We hope to broaden critical discussions on participatory research approaches and to support new and established participatory research practitioners.

\section{Approach}

In November 2017, we met as a group of women researchers from diverse disciplines and settings in Durham in North East England to discuss some of the complex experiences, difficulties and risks we had experienced in participatory research. Our commonalities were that we all use participatory research in our core research practice focused on social justice issues. We used a participatory process in our discussions and collaborative writing. Caroline documented our reflections and responses to examples from practice that each of us provided in four non-prescriptive categories (the themes discussed here) to guide the writing phase. To ensure that our diverse and subjective experiences transpired in our collaborative writing, we were committed to avoiding homogenizing our many voices, as reflected in our different writing styles when describing the themes. The brief was to focus on difficulties encountered, rather than to recount our research projects in detail, but we refer to contextual specificities wherever possible.

We comprise a mix of early, mid- and late-career academic researcherpractitioners. We all undertake participatory research in one (or more) of three locations, namely Australia, the UK and Hong Kong. Because we are at different career stages, some of us have already published on this topic, while for others, this is the first opportunity to critically reflect on the participatory research process. We acknowledge the importance of our individuality and sociocultural frames of reference in discussing and writing about these issues, particularly from our positions as women researchers from diverse ethnic and language backgrounds.

We also have diverse understandings of what participatory research means, and different participatory research practices. Yet, we identified common experiences, irrespective of discipline, career stage, background and geographical location. We acknowledge that the term 'participatory research' is used in many different ways and has many strands and histories (see Abma et al., 2019: 7-13). The extent to which community-based researchers co-create all elements of the research design, process and outcomes also varies, with some projects initiated as community-university partnerships, while others may be initiated by academics and have elements of participatory methods. We also acknowledge the complex and problematic notion of 'community' (see Weston and Lenette, 2016). A distinction is often made between participatory research as a holistic approach based within a participatory paradigm, and the use of participatory methods (such as photovoice or participatory mapping) in more traditional research projects (see Banks and Brydon-Miller, 2019: 17). Often, the use of participatory methods may lead to a piece of research or a research partnership becoming more participatory in other respects. 
We present a discussion of four themes representing key difficulties and risks we encounter in participatory research: (1) participatory research as a gendered field; (2) navigating the grey zone; (3) complex relationships among co-researchers; and (4) negotiating frameworks for practice. We then turn to the literature on two broader research issues, namely the exclusion of content on complex research processes in academic publications, and navigating the intricacies of power differentials, to position our reflections in relation to current debates.

\section{Theme 1: Participatory research as a gendered field}

Our reflections on participatory research as a research methodology, and our past experiences, prompted the observation that a high proportion of researchers who undertake participatory research are women. Participatory research is often seen to require more work (see Pain, 2004), is more emotionally taxing (Alexandra, 2017), and can at times involve more risky situations (with the potential to compromise researchers' well-being) than non-participatory methods (Dickens and Butcher, 2016); so why are participatory research researchers disproportionately women? We are not alone in pondering on this state of affairs; Pain (2004: 659), for instance, concludes: 'That the practice of PR [participatory research] is gendered is at the heart of this [marginalization of participatory research approaches]; women and feminist geographers predominate.'

Participatory research as a sensitive and appropriate tool to uncover gendered perspectives in collaboration with community-based researchers is discussed at length in the literature (Lenette, 2017; Mclntyre, 2003), but implications surrounding the impact of the gender of researchers themselves is less well documented. From personal observations and anecdotal evidence, we came to the realization that the fields in which participatory research tends to dominate (such as cultural geography, education, disability, social movements, and migration studies) are themselves gendered. Furthermore, community-based researchers who tend to gravitate towards participatory research methods are also disproportionately women. As Fields (2016: 32) notes, the 'embodied experiences' of race, gender, sexuality and class mark the research process at every stage, especially in PR where the construction of meaningful relationships is at the heart of projects, and so this lack of consideration of the gendered nature of the field is striking. Relatedly, Caretta and Riaño (2016: 260) noted that in participatory research, the "'co" of co-production, co-determination, and collaboration can engender friction and strain'.

Women researchers (both academic and community-based) in participatory research projects face challenges related to gender and sexuality. Several of us reflected that research relationships featured complex and nuanced interactions where we tried to balance friendships, research and collaboration amid gendered and sexualized interactions. For instance, one co-author reflected on her decision to accompany a community-based researcher, a young male asylum seeker, on an excursion late at night: what expectations might he bring to the situation? While all of us take seriously the discomfort and potential danger in terms of physical safety of such situations (particularly as lone women academic researchers), these tense moments can also become important empirical vantage points to gather evidence from experiences for further reflection on our approach. But it can be difficult to consider the implications of expressing that discomfort candidly to community-based researchers without feeling that the research relationship might be jeopardized. This raises very different issues in comparison with traditional qualitative researchers' relationships with their research 'informants', with whom they might expect to have a more protective role. 
Thus, the gendered nature of participatory research not only creates new avenues for risk and reflection, but also orientates the outcomes of research in particular ways. Researchers have noted the alignment between participatory research and feminist research goals prioritizing care, empowerment and social justice (Maguire, 2001), even as participatory researchers themselves do not always articulate such connections directly (Gatenby and Humphries, 2000). Yet, the reparative impulse guiding much participatory research is itself gendered, often emerging from personal relationships forged between academic and community-based researchers. For example, this reparative impulse underscored Alexandra's (2017: 340) reflections in her field notes on a particular incident in the context of a media production project with communitybased researchers who were newly arrived people seeking asylum in Ireland:

I am also worried. I want to hug Ahmad - to hug someone in pain, someone who I have come to know and respect, seems the 'right' response ... Of course, I am not Ahmad's friend in the traditional sense of the word, but I have come to know him, and I do not know what to do. I feel responsible, and implicated in this practice.

The porous boundaries described in this reflection from a woman academic researcher in relation to a male community-based researcher encompass worry, affection, empathy, and feeling responsible and implicated. The drive to conduct participatory research that "lays bare and learns from moments of difficulty" involves greater emotional risks for all involved' (Fields, 2016: 37).

We note from experience the extent to which our research has put us at greater risk of exposure to harm. One co-author intervened in a situation of domestic violence where a community-based researcher, a young woman fearing for her life, asked for help while in a very precarious situation. She was welcomed at the co-author's home overnight, and shared details of her distressing circumstances (the research topic was not domestic violence). The author believed that such interventions were 'normal' in the context of developing trust as a participatory researcher, and that welcoming this young woman in her home was more important than thinking about the implications for the author's own safety. When the young woman decided to return to her home and subsequently never mentioned this incident again, it became very difficult for the researcher to simply 'forget' the safety issues and continue with the research. Both parties decided to cease their collaboration. As a result of this incident, the co-author suffered emotionally and physically from vicarious trauma. Such situations also arise in traditional qualitative research, where research informants may be in danger.

Especially because of its gendered dimensions, institutions and disciplinary conventions do not always value participatory research. Mountz et al. (2015: 1,242) conclude that academic work focused on topics such as care, social justice and social reproduction or using participatory research methods has typically been feminized and undervalued. Participatory research projects that are 'ontologically, epistemologically and methodologically grounded in emancipatory goals' continue to 'create friction with a social science that continues to be dominated by a positivist, conservative scientific paradigm' (Duckett and Pratt, 2001: 832). As measurements of impact dominate evaluation of scholarship in Australia and the UK, the uncertain or subjective outcomes, processual nature of the work, and difficulty in measuring impact undercut the potential value of participatory research approaches in many disciplines. The perception of reparative work as 'feminine' must also be understood as part of the devaluing of participatory research approaches (Mountz et al., 2015). Consequently, researchers should take up every opportunity to discuss the gendered nature of 
participatory research, so that it remains central to debates about what counts as legitimate or important academic practice and knowledge.

\section{Theme 2: Navigating the grey zone}

The dynamic and relational nature of participatory research means that there is seldom a 'right' way of proceeding. Frequently, we are navigating shifting - and competing - opportunities, risks and agendas, with ramifications both for the research and for collaborators. While other researchers, notably ethnographers (Murphy and Dingwell, 2007), often work in a similarly grey zone (that is, spaces where processes and relations are not always clear-cut), these challenges are complicated in participatory research by the complex nature of research relationships, where community-based researchers may sometimes be seen as both 'informants' and 'collaborators' from academic researchers' perspectives. Unpacking the experiences of two co-authors, we explore dimensions of this grey zone, namely 'mapping the boundaries of the research' and 'negotiating democracy in cultural hierarchy'.

\section{Mapping the boundaries of the research}

With academic and community-based researchers engaging in a range of formal and informal encounters - often engendering a sense of trust and intimacy - agreeing on what constitutes data can be challenging. Inevitably, we come to 'know' more than we can capture, but how do we decide what should be captured? And who decides? In an arts-based study involving a co-author, the boundary was rendered visible in one instance when a community-based researcher shared information with an artistresearcher on the explicit proviso that it was not recorded in her field notes or shared with the academic researcher. For another co-author, 'out-group' disclosures that offered insights for solving 'in-group' problems in a project required the renegotiation of disclosure and anonymity to secure trust and maintain confidentiality. Both scenarios demonstrate the porosity of research boundaries, and the challenges this presents for negotiating if and how data are to be captured and used. While the boundaries may initially be established in collaboration agreements and consent processes, even the most flexible and iterative of these are not malleable enough to adjust to the multiple shifts in relations, contexts and focuses that can occur during participatory research. Approaches to managing this may include:

1. Setting firm boundaries that exclude certain forms or sites of encounter (for example, communication outside of formal sessions). This might be a useful guideline for community-based researchers, or, conversely, lead them to secondguess whether they can or should share uncomfortable but important information outside of 'research time'. Academic researchers, on the other hand, may miss opportunities to uncover valuable information and insights.

2. Capturing everything, on the proviso that consent has been granted. However, this approach risks perceived breaches of trust and potential regret if information is shared in moments of forgetfulness when a sense of intimacy transcends the research relationship (Mayan and Daum, 2016).

3. Negotiating on a case-by-case basis, either on the spot or prior to use in analysis/ publication, where reciprocal benefits are clear for community-based and academic researchers.

Nonetheless, such negotiations risk including/excluding data based on levels of trust established at particular points in the project, and on community-based researchers' 
understandings of the research process and outcomes, which inevitably develop over time. These issues are relevant to all forms of research, but are pertinent to participatory research given the strong agenda of knowledge co-creation. Seeking additional consent to incorporate information communicated outside the project 'boundaries', either through field notes or by introducing the topic into the formal project space, is one way to address this tension. In such cases, however, additional caveats relating to anonymity and/or audience may be required. In particular, community-based researchers are often more open to sharing sensitive material with academic researchers than within their own networks, where the personal stakes are perceived to be much higher. To paraphrase a community-based researcher's reflection in one project: 'I want you to know, but don't tell my community.' Where consent is not gained to incorporate relevant information, a different challenge emerges: the challenge of unknowing. Yet, such unknowing is inevitably limited, and this information may still - consciously or unconsciously - inform data analysis and selection of material for publication (see Hugman et al., 2011, for their discussion of this as an intentional process).

\section{Negotiating democracy in cultural hierarchy through partnership}

Participatory research attempts to promote democracy and equality among everyone involved in carrying out the research (Kara, 2017). But the literature frequently overlooks the intricacies of relationships among co-researchers (academic and communitybased) in the co-creation of knowledge, despite how 'muddled' (Mayan and Daum, 2016: 69) such relationships can become in participatory research projects. Because these notions are themselves culturally informed, practising participatory research in cultures where hierarchical collectivism prevails inevitably highlights the need for cultural negotiation (Brannelly and Boulton, 2017), especially when community-based researchers have pre-established hierarchical relationships among themselves before they participate in research projects, as illustrated in the examples below.

In a co-author's participatory action research project in Hong Kong with participants from Chinese ethnicities, democratic practices promoted within the inquiry group disrupted pre-existing and culturally valued familial hierarchy (see Ho, Jackson and Kong, 2018). Referring to friends and neighbours as 'sister/brother' or 'mother/father' can legitimize care obligations towards each other, particularly from junior to senior persons in the hierarchy. Promoting egalitarian communication or relations among community-based researchers who are situated in familial hierarchy can create threats to their sense of moral integrity (ibid.). For example, disagreeing with a mother figure in the inquiry group can be considered a violation of the Chinese virtue of 'filial piety', and can convey a lack of gratitude (Kong, 2014; Kong and Hooper, 2018). However, without transforming the pre-established hierarchy, community-based researchers who are 'junior' in the hierarchy could be prohibited from speaking up in the group. These experiences question the Eurocentric understanding of 'democracy' (Ho, Jackson and Kong, 2018), and point to the need to make sense of how 'democracy' could be understood and negotiated in the everyday life practices of people, in this case a Chinese community, which may emphasize 'hierarchical harmony' over 'nonhierarchical dialogue' (Ho, Kong and Huang, 2018).

Intersectionality of culture and gender adds another layer of complexity to navigating the cultural space for democratic practices. Another co-author experienced similar issues in her participatory research project, in relation to cultural gender norms. When researching alongside young people from refugee backgrounds recently settled in the UK, she came to understand that culturally embedded gender roles and relations had been mediating the form and content of interactions and contributions. In this 
participatory arts-based research, the co-author encountered male dominance within the cohort due to emerging gender politics in the young people's ethnic community. It became increasingly clear to the researcher that the young women were circumscribing their own involvement in aspects of the project. While outspoken and creatively brave in the project space, they were unwilling to enact this publicly, notably electing not to sing the songs they had created in front of an audience. This was due to concerns about how male adult community members would respond, and the implications for their own and their parents' reputations.

Ultimately, the challenge that participatory researchers face is the displacement of the centrality of traditional 'Western-influenced' ways of constructing knowledge in their research encounters. Barnes et al. (2017) suggest that we should translate the cultural, social and spiritual concerns into methodological approaches for capturing 'non-Western' ways of knowing. They emphasize the importance of partnerships for acknowledging each other's cultural positionality, which can transcend the 'your/ my culture' dichotomy to creatively find ways to incorporate both. Partnerships in participatory research are also processes of mutual inquiry into each other's cultural practices, revealing the multiplicity of cultures that each person values, and avoiding cultural essentialism. The notion of partnership speaks to the importance of the ethics of care (Banks et al., 2013; Brannelly and Boulton, 2017) and dialogue (Feyerabend, 1991) in participatory research as useful tools to navigate issues such as those raised here. In the examples outlined above, negotiating democracy in cultural hierarchy was achieved by cultivating a family-like community of practice that retained the filial piety valued in Chinese culture, valuing disagreements among community-based researchers, supporting the women to chart their own course through the complexities of self-expression and community politics, and fostering equitable and respectful relations among young people within the research project space. Instead of imposing a unilateral notion of democracy on community-based researchers, creating spaces for confronting differences and disagreement in a 'careful' way (Kara, 2017) seems to be more sensitive and effective in meaningfully localizing democratic practices.

\section{Theme 3: Complex relationships among co-researchers}

In an increasingly restricted funding landscape, partnerships between universitybased and community-based researchers can facilitate embodied situated knowledge production, as well as create pathways for public engagement and social change. As Facer and Enright (2016: 64) note, such partnerships face 'complex webs of accountability' driven by multiple, not always shared, goals. Embracing ethical principles, a shared vision and understanding of research aims and objectives, and clear organizational frameworks and processes are necessary prerequisites for ethical participatory research (Centre for Social Justice and Community Action and NCCPE, 2012). The examples below highlight two such dimensions, namely 'negotiating partnerships' and 'everyday ethical dilemmas'.

\section{Negotiating partnerships}

Issues of power imbalances and the importance of establishing equitable relationships have been addressed by many researchers collaborating with community-based researchers and partners. Strategies for academic researchers to gain access via gatekeepers, as well as issues of positionality, have also been examined extensively (Clark and Sinclair, 2008; Corra and Willer, 2002; Yancey et al., 2006; Yu, 2009). Nevertheless, as McAreavey and Das (2013: 114) point out, there has been limited literature on 'real 
life ethical dilemmas' encountered in participatory research when engaging with community-based researchers and partners on an everyday level, although this is now changing (see Banks et al., 2013; Banks and Brydon-Miller, 2019; Lenette et al., 2013). A key issue is that community gatekeepers acquire an important role in social research, as they hold power to allow or deny access to a particular community or institution (De Laine, 2000). As Clark (2011) reminds us, research processes can become complex, as gatekeepers often serve as cultural mediators or 'brokers', vouching for academic researchers' credibility, and consequently influencing recruitment and retention (see also Oka and Shaw, 2000; De Laine, 2000).

Furthermore, working in partnership with organizations and communities involves establishing long-term communication avenues that allow dialogue and knowledge exchange, and are transparent. According to Banks et al. (2013), working in partnership adds further complexity when considering ownership of data and findings for dissemination, suggesting that partnership is an ongoing process that is constantly under review. As such, all members of a research team must be attuned and responsive to varying degrees of leadership required during different research stages (Gillis and Jackson, 2002). Despite the image of participatory research as a more egalitarian model of research, power hierarchies are inherent to research processes, and academic researchers in particular may often find themselves in a matrix of competing hierarchies and expectations (for example, stemming from the community, co-researchers and colleagues, the institutions or themselves) when working in this way.

\section{Everyday ethical dilemmas}

Drawing from personal experiences of participatory research, two co-authors share their experiences of having to negotiate different perspectives, interests and power imbalances in two distinct studies in the UK. One experienced co-author recalled how she wielded power reluctantly to enforce a contract between two parties:

I worked with a small local community organization and larger national NGO in a community-university research partnership on a funded twoyear action research project. We knew each other from previous work together, co-designed the research project and submitted the funding bid as partners. We had a partnership agreement, which outlined the responsibilities and funding allocated to each partner. The university was the fund-holder and I was the 'principal investigator', hence ultimately responsible for reporting progress and ensuring the project kept on track. The community organization survived through short-term grants to undertake specific pieces of work, and so the research grant was vital to maintain the salary of the existing community organizer. We started the project before recruiting a university researcher, as the community organization needed the money to keep the staff member in post. During the first few months of the project, the community organizer did little work on the project, instead working on other pieces of short-term funded work. The national NGO worker also played a minimal role in co-managing the project. After several meetings and unmet promises to undertake more work, I reluctantly decided to use my power to ask the university finance department to notify our partners that they could not claim the next quarter's funding until they had delivered more of the promised work. At this point, I was glad about the university's requirement to set up a detailed agreement specifying the work to be done by each partner, even though 
at the time it seemed too tying for participatory research. I felt bad about initiating what was, in fact, a threat to withhold funding. But I realized I was being exploited - having to pick up much of the extra work myself.

In another case, an early career academic researcher explains how she had no choice but to challenge a community partner's wishes to proceed with her project:

In an arts-based participatory ethnographic study in the UK, I found myself in the precarious position of having to manage expectations beyond the research outcomes, as the potential community partner requested that part of the project's audiovisual data should be used as online marketing material for the organization's website. Negotiating partnership with this organization was a multiphased process as, initially, the organization agreed to be involved in the project and then contacted me with 'recommendations' on producing video testimonials, which resulted in a six-month email exchange and delayed start date. I found myself in a critical position, having to decide between 'jeopardizing' community access by declining to adopt the partner organization's suggestions, or sacrificing the project's academic integrity. As an alternative, I collaborated directly with community-based researchers, making contacts through snowball methods, to ensure they could decide the project's arts-based activities and outputs.

Our role and competency as academic participatory researchers rest on our networks and relationships with community-based researchers, communities and partners, and so our investment in projects and our relationships with community members, who are often ignored and marginalized, does not always allow space for 'pushing back'. As these examples highlight, we agree with McAreavey and Das (2013) that participatory research inherently involves a 'delicate balancing act' to negotiate specific partnership rules, while safeguarding the integrity and academic rigour of the research.

\section{Theme 4: Negotiating frameworks for practice}

The context within which we work, including institutions, organizations and community networks, are not value-free. Each is driven by their own set of values and nested in wider ecosystems. Whether those wider ecosystems reflect the same values or not will necessarily impact on communities. Participatory research is an explicitly value-driven approach, and so navigating various systems for funding and supporting research can present a number of challenges. To find a route through the various systems, we tend to work on two fronts: (1) to foster improved recognition and understandings of participatory research, which includes working towards having the quality, purpose and processes of our work explicitly recognized (Cook, 2012; ICPHR, 2013); and (2) more pragmatically, camouflaging our work under the mantle of other value systems. In safe places, such as in meetings with other participatory researchers, we discuss the challenges we encounter and confess our 'guilty secrets' (Cook, 1998) that this range of challenges sometimes leads us to struggle with the very essence of our work.

\section{The hierarchy of methodology, method and research funding}

In health research, for example, there is a common mantra about research of 'gold standard'. This does not mean, as we might think, the best research of its kind, but research that adheres to linear models that achieve predicted outcomes within certain, 
predetermined contexts (particularly in randomized controlled trials). Our challenges as participatory researchers are how to get research funded if we are honest: for example, if we say that we know the general issue to be researched but not what the specific question might be until we begin the critical reflection inherent to our research process; if we say that we are not sure what the best methods are yet, as this will emerge as we develop our work together; if we say that we are all researchers, so 'participants' is not a relevant concept; and if we say that, while we have some hopes and expectations for outcomes, others will be generated as the research process develops. Consequently, the processes of collaboration, and the way in which research evolves in participatory research, are in danger of being categorized negatively. The recursive, relational approaches to generating and validating forms of knowing that have an impact on the community of practice involved are not universally recognized as method. For many of us, our guilty secret is that we use illusory consensus as a screen. For instance, to make our case for funding more acceptable, we might use accepted methodological terminology and name the communicative spaces in our research 'focus groups', and in that way we can fly under the radar and secure funding. We can, and do, do this, but we carry the burden of that artifice. Our burden is not that we believe we are being unethical, carrying out research that lacks rigour or failing to understand what is needed, but rather the knowledge that what we are doing is ethical, rigorous and methodologically sound. We have not been able to articulate that, however, within the confines of another framework (that is, institutional) for scrutiny. Our guilty secret is then that, to get funding, we have denied our discipline the right to be judged on its own merits, since we suspect that because of the usual framework for scrutiny, this would jeopardize its chances of being funded.

\section{Recognizing the effects of the 'impact agenda'}

In recent years, there has been increased awareness of the need for applied research to go beyond being predominantly a tool for knowledge collection, and to make a difference to communities - that is, to have impact. While often difficult to measure in participatory research, impact is now central to the metrics of success for many universities. In this context, we might expect that research such as participatory research, which has an explicit intention for transformative action, would be valued. While impact here can be conceptualized as the beneficial changes that happen in communities as a consequence of participation in research, common impact metrics privilege the tangible, quantifiable and global, rather than the subjective, qualitative and local changes that may be more subtle, complex and difficult to capture and articulate. The issue is summarized by Pain et al. (2015: 4) as:

... the attempt to measure 'impact' as a concrete, visible phenomenon that is fixed in time and space, that one party does to another party ... whereas deep co-production is a process often involving a gradual, porous and diffuse series of changes undertaken collaboratively that may be demonstrable but not always measurable.

We recognize that, when we define the impact of participatory research within current university frameworks, the fundamental embedded, transformational process and changes that emanate from participatory research are at best undervalued and are most likely lost in reports of our research. 


\section{The impact of institutional requirements on participatory researchers}

If we are to reach audiences beyond academia, we need to write in practitioner journals, local newsletters and social media. Such outlets are not, however, routinely valued in terms of academic impact measurements. In the UK, for example, there is a set of standards for assessing the quality of research in higher education institutions determined by a process known as the Research Excellence Framework (REF) (www.ref.ac.uk). One measure used in this process is the number of publications in refereed journals, preferably with high impact factors (a measure of the yearly average number of citations to articles published in a journal). To publish in journals with lower (or no) impact factors leaves academic researchers open to institutional pressures that can affect our status in terms of performance measures, career opportunities and well-being. Academic researchers can thus be torn between project requirements and university commercial imperatives that determine how our time is allocated, and how the use of our time is judged.

The timeframes for participatory research can also challenge institutional expectations and requirements. The 'long time involved in conducting communitybased research presents challenges not only in working with funding agencies, but also with the shorter-term expectations typical of ... universities' (Israel et al., 1998: 192). Short timeframes for research can disadvantage participatory research approaches that start from the gradual building of relationships. This is especially so where people experience social marginalization, and might need longer to become involved in any form of research. A consequence of the lack of time is that academic researchers engaged in participatory research often use their own time to develop research (Abma et al., 2019). Despite such a personal commitment to building research possibilities, those of us who work in academic institutions have found that our research remains marginalized, as participatory research challenges predominant frameworks about what constitutes 'quality' in research (Cook, 2012). We find that it is less likely to fit the REF measures in the UK, and the associated institutional standards created in line with perceptions of what is needed for REF. An example of this is how academics in the UK and Australia are being judged by the amount of research money they successfully bid for; the scale of their research (with large, multisite, international research being more highly valued than locally transformative long-term engagements); and how many citations are recorded per output (Chubb et al., 2017). Commissioned to review the UK REF, Lord Stern (2016: 14) noted how it can drive researchers 'towards safe topics and short-termism, and a reluctance to engage in risky or multidisciplinary projects, in order to ensure reliable, high quality publication within the REF period', and that it 'may be discouraging innovative thinking and risk taking'. This has obvious implications for participatory research.

These research frameworks and metrics create barriers to building local participatory research programmes, especially with people who are marginalized. The lack of recognition of co-researcher models is both a methodological and a social justice issue. It is therefore vital that the research community overall revisits, reexamines and revises the marginalization of participatory research and its proponents, or what Sushama et al. (2018: 6) termed the 'traditional scientific conventions that bound [studies] in terms of time, money and scope'. This needs to be done with recognition of the purposes, processes and quality of participatory research forms, and those who are in a position to do so should speak out about the methodology. Only if all aspects of our work are honestly revealed can these be explored and collated 
to support the development of a body of knowledge in relation to the emancipatory impact of participation and the opportunities for creating knowledge for change.

\section{Discussion}

The themes discussed here are by no means the only issues emerging from our participatory research experiences and discussions, but they are prominent issues through which we hope to launch an open discussion on the topic, so that others will continue to build on our findings and contribute reflections on their own experiences, dilemmas and strategies to the literature. Through our writing, we want to reach out to other academic researchers, particularly those who may feel frustrated, guilty or confused about the problematic aspects of participatory research, and invite them to engage in a reflexive process. When talking about ethical issues in research, Guillemin and Gillam (2004: 265) comment: 'We need both a language to articulate and understand ... ethical issues and an approach that assists us to deal with these issues when they arise.' We share our strategies as part of the reflections that may assist academic researchers to tackle some of the challenges of participatory research, while resisting the temptation to offer definitive 'solutions' that might constrain its heuristic endeavour.

We value participatory research as a mode of inquiry that focuses on gaining knowledge through action-based processes that use a 'bottom-up approach' to challenge conventional hierarchical structures of research environments (Cornwall and Jewkes, 1995). We subscribe to the view that participatory research can be used to challenge one or several sociopolitical inequities (Mayan and Daum, 2016). Our understanding is that 'we work closely with one another, as equals, by negotiating roles, paying attention to reciprocity, and working to develop trust' (ibid.: 72; see also Centre for Social Justice and Community Action and NCCPE, 2012; Banks et al., 2013). As such, participatory research methods promote multi-vocality and are invested in supporting the democratization of knowledge production through working with community-based researchers (both individuals and organizations) to create a safe and inclusive research environment that addresses community needs and involves both individual and collective voices in the interpretive process (Wallwork, 2002).

We identified two key issues that frame our reflections in relation to current debates. The first concerns our un/conscious decisions to exclude content on research processes, especially the more problematic or difficult to articulate themes. The second relates to how we manage a range of sociocultural complexities in our research practice, but particularly the intricacies of power differentials.

\section{Exclusion of content on participatory research processes in academic publications}

Our experience of getting our work published has been mixed. Attempting to publish in journals based on subject area, as opposed to social research methods, can prove difficult. Journals, like research funders, have historical frameworks for recognizing rigour based on particular paradigmatic understandings. Knowing this, many of us publish in journals where our approach to research has been championed or at least accepted. This limits our reach and impact and creates a 'preaching to the converted' effect. Impact that occurs indirectly through non-linear mechanisms thus remains underrepresented in published accounts of research evidence (Greenhalgh and Fahy, 2015).

Furthermore, the literature acknowledges that research involving deep relationships of trust with community-based researchers needs to be managed 
carefully, and can be challenging and distressing at times for all concerned (Cook, 2012; Banks et al., 2013; Foster, 2016; Rasool, 2018). Yet, the more textured and difficult aspects of participatory research may be deliberately excluded - usually through selfcensorship - from traditional avenues such as peer-reviewed academic journals, due to perceptions that the focus should be solely on disseminating positive findings and 'victory narratives' (Owen et al., 2005: 339), rather than on reflexive research practice where difficulties are highlighted. Wright et al. (2012: 45) also agree that 'the "behind-the-scenes" emotional work of preparing for research, building relationships and rapport with others, thinking, conversing, and representing is typically removed from conventional academic accounts'. The 'hierarchy' of content that authors are supposed to include/exclude privileges the more straightforward 'steps' undertaken in participatory research (for example, methodology, findings, discussion and implications), to the detriment of content on emotionally challenging or risky aspects of the research, such as the examples we have shared in this paper. As such, when boundaries become blurred in the field and impact on decision making, there is a lack of space to discuss such tensions openly. For instance, when academic researchers feel obliged to engage in a range of activities or obligations that resemble 'close' friendships, such as lending money, driving community members or taking calls at weekends (see MacFarlane et al., 2019; Mayan and Daum, 2016), or when research relationships come to an end and feelings of guilt and frustration emerge (Cox et al., 2014), academic researchers may be reticent to discuss such issues openly in publications. We argue that it is, in fact, crucial to identify, reflect upon and address such issues at interpersonal and broader levels in traditional and creative academic outputs to broaden debates and scholarly discussions on the realities of fieldwork and research frameworks more generally.

\section{Power differentials}

The defusing of power differentials is an intrinsic aim of participatory research, which aims to prompt equitable participation and dialogues among co-researchers (Kara, 2017). Unsurprisingly, this issue emerged through our accounts as a key tension to navigate, as academic 'researchers must grapple with power and vulnerability - both those of other people as well as their own' (Dodson et al., 2007: 822). To reduce power imbalances between 'powerful' academics and 'vulnerable' community-based researchers, institutional ethics clearance procedures aim to limit the potential for abuse of power. But in the face of unforeseen dilemmas and risks in participatory research, academic researchers are often 'on their own' when deciding on the best course of action (Lenette et al., 2018; Blake, 2007). In this context, collaboratively balancing expectations and requests without compromising one's academic integrity is not unusual, reflecting the 'emotional labor' (Foster, 2016) involved in participatory research. Conversely, as we explore in our reflections, virtues of care, compassion and equality upheld by many academic researchers engaged in participatory research can also become their 'burdens', sometimes even putting them in 'dangerous situations' where they can get hurt (Banks et al., 2013; Kara, 2017). Thus, we argue that telling stories about how academic researchers navigate intricate power differentials in the field, and in the broader context of research, with varying degrees of success, and acknowledging situations where lack of reflexivity may have resulted in detrimental outcomes, would further enrich discussions on participatory research. 


\section{Conclusion}

Our group discussion on the risky aspects of participatory research has reinvigorated our commitment to the approach rather than dampened it. We concur with Mayan and Daum (2016: 74) that 'if achieving great things means living in a more equitable and just society, we must take the risks that come with engaging in CBPR [communitybased participatory research]'. We engage in this type of research precisely because of shared concerns for socio-economic inequities, and our wish to challenge such situations through participatory research, and our commitment to democratizing research spaces and knowledge. We value the work of others in this space, such as the International Collaboration for Participatory Health Research (see ICPHR, 2013), and scholars such as Walmsley and Johnson (2003) and Nind (2014) on inclusive research approaches.

We must continue to promote meaningful engagement and collaborations in our participatory research practice, and opening up a space for candid, honest conversations about difficult and risky aspects can contribute to achieving this aim. We remain acutely aware that participatory research is not 'magical' just because it is participatory, nor is it appropriate in all contexts and in all collaborations. Our aim is not to state that our distinct approaches are 'right' or 'wrong'. Rather, what brings us together as an alliance of participatory researchers is our common experience of navigating moments of complexity, crisis and doubt with few (academic) resources to guide us.

We offer these reflections, and related strategies, as a contribution to critical debates on participatory research practice, and as a provocation to others across disciplines and geographical locations to similarly share their challenges and strategies. We do so in the hope of fostering further interdisciplinary debates across contexts, so that others will join us and add their own experiences, frustrations and strategies to this conversation. Difficulties and risks are contextually specific and take many forms, and so we would welcome the opportunity of extending our own knowledge on how these can manifest in others' research practices. We invite academic and community-based researchers to engage in reflections on the difficult and risky aspects of participatory research with trusted colleagues and using meaningful processes, and to share unique perspectives with us and others to keep this conversation and reflexive endeavour alive. We hope that in so doing, the plurality of perspectives that can be documented and debated will further enrich our understanding of participatory research as a distinctive approach.

\section{Acknowledgements}

We acknowledge the Durham Senior Research COFUND Fellowship (so named because it was cofunded by Durham University and the European Union, and Durham University's Centre for Social Justice and Community Action) for providing the intellectual environment for this paper.

\section{Notes on the contributors}

Caroline Lenette's work focuses on arts-based methods in participatory research with people from refugee and asylum seeker backgrounds, particularly refugee women. She explores the links among creative methods, ethics and policy. 
Sarah Banks works on professional ethics in social, community and youth work; ethics in community-based participatory research; participatory methodologies; and approaches to promoting equity and social justice.

Caitlin Nunn's research engages with the lived experiences of refugees, with a particular focus on youth, resettlement and belonging. Much of her work involves collaborative and arts-based approaches.

Kate Coddington's work focuses on borders, migration and postcolonial policymaking in the Asia-Pacific, and has included collaborations with non-governmental and advocacy organizations.

Tina Cook's research focuses on democratic approaches to research and practice. She has a particular interest in participatory action research as a means for facilitating the inclusion, as research partners, of those who might generally be excluded from research that concerns their own lives.

Sui Ting Kong is interested in social work practice research, methodological innovation, and theorizing intimate partner violence and practices of intimacy. Her work looks at how to collaborate with separated abused women for providing post-separation care and support.

Nelli Stavropoulou explores the transformative role of participatory arts research as a vehicle for self-expression for individuals seeking asylum. Her work combines visual methods (photography and film-making) with participatory action research principles.

\section{References}

Abma, T., Banks, S., Cook, T., Dias, S., Madsen, W., Springett, J. and Wright, M.T. (2019) Participatory Research for Health and Social Well-Being. Cham: Springer.

Alexandra, D. (2017) 'Implicating practice: Engaged scholarship through co-creative media'. In Jamissen, G., Hardy, P., Nordkvelle, Y. and Pleasants, H. (eds) Digital Storytelling in Higher Education: International perspectives. Cham: Palgrave Macmillan, 335-53.

Banks, S., Armstrong, A., Carter, K., Graham, H., Hayward, P., Henry, A., Holland, T., Holmes, C., Lee, A., McNulty, A., Moore, N., Nayling, N., Stokoe, A. and Strachan, A. (2013) 'Everyday ethics in community-based participatory research'. Contemporary Social Science, 8 (3), 263-77.

Banks, S. and Brydon-Miller, M. (2019) 'Ethics in participatory research'. In Banks, S. and BrydonMiller, M. (eds) Ethics in Participatory Research for Health and Social Well-Being: Cases and commentaries. London: Routledge, 1-30.

Barnes, H.M., Gunn, T.R., Barnes, A.M., Muriwai, E., Wetherell, M. and McCreanor, T. (2017) 'Feeling and spirit: Developing an indigenous wairua approach to research'. Qualitative Research, 17 (3), 313-25.

Blake, M.K. (2007) 'Formality and friendship: Research ethics review and participatory action research'. ACME: An International e-Journal for Critical Geographies, 6 (3), 411-21.

Brannelly, T. and Boulton, A. (2017) 'The ethics of care and transformational research practices in Aotearoa New Zealand'. Qualitative Research, 17 (3), 340-50.

Caretta, M.A. and Riaño, Y. (2016) 'Feminist participatory methodologies in geography: Creating spaces of inclusion'. Qualitative Research, 16 (3), 258-66.

Centre for Social Justice and Community Action and NCCPE (National Co-ordinating Centre for Public Engagement) (2012) Community-Based Participatory Research: A guide to ethical principles and practice. Durham: Durham University. Online. https://tinyurl.com/y3q4shsv (accessed 2 June 2019).

Chubb, J., Watermeyer, R. and Wakeling, P. (2017) 'Fear and loathing in the academy? The role of emotion in response to an impact agenda in the UK and Australia'. Higher Education Research and Development, 36 (3), 555-68.

Clark, T. (2011) 'Gaining and maintaining access: Exploring the mechanisms that support and challenge the relationship between gatekeepers and researchers'. Qualitative Social Work, 10 (4), 485-502. 
Clark, T. and Sinclair, R. (2008) 'The costs and benefits of acting as a research site'. Evidence and Policy: A Journal of Research, Debate and Practice, 4 (1), 105-19.

Cook, T. (1998) 'The importance of mess in action research'. Educational Action Research, $6(1), 93-109$.

Cook, T. (2012) 'Where participatory approaches meet pragmatism in funded (health) research: The challenge of finding meaningful spaces'. Forum: Qualitative Social Research, 13 (1), Article 18. Online. https://tinyurl.com/clspoua (accessed 2 June 2019).

Cook, T., Boote, J., Buckley, N., Vougioukalou, S. and Wright, M. (2017) 'Accessing participatory research impact and legacy: Developing the evidence base for participatory approaches in health research'. Educational Action Research, 25 (4), 473-88.

Cornwall, A. and Jewkes, R. (1995) 'What is participatory research?'. Social Science and Medicine, 41 (12), 1667-76.

Corra, M. and Willer, D. (2002) 'The gatekeeper'. Sociological Theory, 20 (2), 180-207.

Cox, S., Drew, S., Guillemin, M., Howell, C., Warr, D. and Waycott, J. (2014) Guidelines for Ethical Visual Research Methods. Melbourne: University of Melbourne.

De Laine, M. (2000) Fieldwork, Participation and Practice: Ethics and dilemmas in qualitative research. London: SAGE Publications.

Dickens, L. and Butcher, M. (2016) 'Going public? Re-thinking visibility, ethics and recognition through participatory research praxis'. Transactions of the Institute of British Geographers, 41 (4), 528-40.

Dodson, L., Piatelli, D. and Schmalzbauer, L. (2007) 'Researching inequality through interpretive collaborations: Shifting power and the unspoken contract'. Qualitative Inquiry, 13 (6), 821-43.

Duckett, P.S. and Pratt, R. (2001) 'The researched opinions on research: Visually impaired people and visual impairment research'. Disability and Society, 16 (6), 815-35.

Durham Community Research Team (2011) Community-Based Participatory Research: Ethical challenges. Durham: Centre for Social Justice and Community Action. Online. https://tinyurl. com/y6l24bwt (accessed 2 June 2019).

Facer, K. and Enright, B. (2016) Creating Living Knowledge: The Connected Communities Programme, community-university relationships and the participatory turn in the production of knowledge. Bristol: University of Bristol and AHRC Connected Communities. Online. https://tinyurl.com/yyyao4lc (accessed 2 June 2019).

Feyerabend, P.K. (1991) Three Dialogues on Knowledge. Oxford: Blackwell.

Fields, J. (2016) 'The racialized erotics of participatory research: A queer feminist understanding'. Women's Studies Quarterly, 44 (3-4), 31-50.

Foster, V. (2016) Collaborative Arts-Based Research for Social Justice. London: Routledge.

Gatenby, B. and Humphries, M. (2000) 'Feminist participatory action research: Methodological and ethical issues'. Women's Studies International Forum, 23 (1), 89-105.

Gillis, A. and Jackson, W. (2002) Research for Nurses: Methods and interpretation. Philadelphia: F.A. Davis Company.

Greenhalgh, T. and Fahy, N. (2015) 'Research impact in the community-based health sciences: An analysis of 162 case studies from the 2014 UK Research Excellence Framework'. BMC Medicine, 13, Article 232, 1-12. Online. https://tinyurl.com/y3zkgyql (accessed 25 May 2019).

Guillemin, M. and Gillam, L. (2004) 'Ethics, reflexivity, and "ethically important moments" in research'. Qualitative Inquiry, 10 (2), 261-80.

Ho, P.S.Y., Jackson, S. and Kong, S.S.-T. (2018) 'Speaking against silence: Finding a voice in Hong Kong Chinese families through the Umbrella Movement'. Sociology, 52 (5), 966-82.

Ho, P.S.Y., Kong, S.-T. and Huang, Y.T. (2018) 'Democratising qualitative research methods: Reflections on Hong Kong, Taiwan and China'. Qualitative Social Work, 17 (3), $469-81$.

Hugman, R., Bartolomei, L. and Pittaway, E. (2011) 'Human agency and the meaning of informed consent: Reflections on research with refugees'. Journal of Refugee Studies, 24 (4), 655-71.

ICPHR (International Collaboration for Participatory Health Research) (2013) What is Participatory Health Research? (Position Paper 1). Berlin: International Collaboration for Participatory Health Research. Online. https://tinyurl.com/qygp3y4 (accessed 25 May 2019).

Israel, B.A., Schulz, A.J., Parker, E.A. and Becker, A.B. (1998) 'Review of community-based research: Assessing partnership approaches to improve public health'. Annual Review of Public Health, 19, 173-202

Kara, H. (2017) 'Identity and power in co-produced activist research'. Qualitative Research, 17 (3), 289-301. 
Kong, S.-T. (2014) 'Participatory Development of Post-Separation Domestic Violence Services: A cooperative grounded inquiry with abused women and their teenage sons/daughters in Hong Kong'. Unpublished PhD thesis, University of York.

Kong, S.-T. and Hooper, C.-A. (2018) 'Building a community of practice for transforming "mothering" of abused women into a "mutual care project": A new focus on partnership and mutuality'. British Journal of Social Work, 48 (3), 633-55.

Lenette, C. (2017) 'Using digital storytelling in participatory research with refugee women'. SAGE Research Methods Cases. Online. https://tinyurl.com/y36tvlkr (accessed 25 May 2019).

Lenette, C., Botfield, J.R., Boydell, K., Haire, B., Newman, C.E. and Zwi, A.B. (2018) 'Beyond compliance checking: A situated approach to visual research ethics'. Journal of Bioethical Inquiry, 15 (2), 293-303.

Lenette, C., Brough, M. and Cox, L. (2013) 'Everyday resilience: Narratives of single refugee women with children'. Qualitative Social Work, 12 (5), 637-53.

Liamputtong, P. and Rumbold, J. (2008) 'Knowing differently: Setting the scene'. In Liamputtong, P. and Rumbold, J. (eds) Knowing Differently: Arts-based and collaborative research methods. New York: Nova Science Publishers, 1-23.

McAreavey, R. and Das, C. (2013) 'A delicate balancing act: Negotiating with gatekeepers for ethical research when researching minority communities'. International Journal of Qualitative Methods, $12(1), 113-31$.

MacFarlane, A., Roche, B., Shabangu, P., Wilkinson, C., Cardol, M. and Hynes, G. (2019) 'Blurring the boundaries between researcher and researched, academic and activist'. In Banks, S. and BrydonMiller, M. (eds) Ethics in Participatory Research for Health and Social Well-Being: Cases and commentaries. London: Routledge, 56-79.

Mclntyre, A. (2003) 'Through the eyes of women: Photovoice and participatory research as tools for reimagining place'. Gender, Place and Culture, 10 (1), 47-66.

Maguire, P. (2001) 'Uneven ground: Feminisms and action research'. In Reason, P. and Bradbury, $\mathrm{H}$. (eds) Handbook of Action Research: Participative inquiry and practice. London: SAGE Publications, 59-69.

Mayan, M.J. and Daum, C.H. (2016) 'Worth the risk? Muddled relationships in community-based participatory research'. Qualitative Health Research, 26 (1), 69-76.

Mountz, A., Bonds, A., Mansfield, B., Loyd, J., Hyndman, J., Walton-Roberts, M., Basu, R., Whitson, R., Hawkins, R., Hamilton, T. and Curran, W. (2015) 'For slow scholarship: A feminist politics of resistance through collective action in the neoliberal university'. ACME: An International e-Journal for Critical Geographies, 14 (4), 1235-59.

Murphy, E. and Dingwell, R. (2007) 'Informed consent, anticipatory regulation and ethnographic practice'. Social Science and Medicine, 65 (11), 2223-34.

Nind, M. (2014) What is Inclusive Research? London: Bloomsbury Academic.

Nunn, C. (2017) 'Translations-Generations: Representing and producing migration generations through arts-based research'. Journal of Intercultural Studies, 38 (1), 1-17.

Oka, T. and Shaw, I. (2000) 'Qualitative research in social work'. Online. http://pweb.sophia.ac.jp/ oka/papers/2000/qrsw/ (accessed 26 May 2019).

Owen, J., Cook, T. and Jones, E. (2005) 'Evaluating the early excellence initiative: The relationship between evaluation, performance management and practitioner participation'. Evaluation, 11 (3), 331-49.

Pain, R. (2004) 'Social geography: Participatory research'. Progress in Human Geography, $28(5), 652-63$

Pain, R., Askins, K., Banks, S., Cook, T., Crawford, G., Crookes, L. et al. (2015) Mapping Alternative Impact: Alternative approaches to impact from co-produced research. Durham: Centre for Social Justice and Community Action. Online. http://eprints.gla.ac.uk/115470/1/115470.pdf (accessed 25 June 2019).

Rasool, Z. (2018) 'Emotions in community research'. In Campbell, E., Pahl, K., Pente, E. and Rasool, Z. (eds) Re-Imagining Contested Communities: Connecting Rotherham through research. Bristol: Policy Press, 115-21.

Southby, K. (2017) 'Reflecting on (the challenge of) conducting participatory research as a researchdegree student'. Research for All, 1 (1), 128-42.

Stern, N. (2016) Building on Success and Learning from Experience: An independent review of the Research Excellence Framework. London: Department for Business, Energy and Industrial Strategy. Online. https://tinyurl.com/yc7go4ws (accessed 26 May 2019). 
Sushama, P., Ghergu, C., Meershoek, A., De Witte, L.P., Van Schayck, O.C.P. and Krumeich, A. (2018) 'Dark clouds in co-creation, and their silver linings: Practical challenges we faced in a participatory project in a resource-constrained community in India, and how we overcame (some of) them'. Global Health Action, 11 (1), Article 1421342, 1-7. Online. https://tinyurl.com/ y4loeaww (accessed 26 May 2019).

Wallwork, E. (2002) 'Ethical analysis of group and community rights: Case study review of the "Collaborative Initiative for Research Ethics in Environmental Health"'. Online. https://tinyurl. com/y5kq8c6s (accessed 25 May 2019).

Walmsley, J. and Johnson, K. (2003) Inclusive Research with People with Learning Disabilities: Past, present and futures. London: Jessica Kingsley Publishers.

Weston, D. and Lenette, C. (2016) 'Performing freedom: The role of music-making in creating a community in asylum seeker detention centres'. International Journal of Community Music, 9 (2), 121-34.

Wright, S., Lloyd, K., Suchet-Pearson, S., Burarrwanga, L., Tofa, M. and Bawaka Country (2012) 'Telling stories in, through and with Country: Engaging with indigenous and more-than-human methodologies at Bawaka, NE Australia'. Journal of Cultural Geography, 29 (1), 39-60.

Yancey, A.K., Ortega, A.N. and Kumanyika, S.K. (2006) 'Effective recruitment and retention of minority research participants'. Annual Review of Public Health, 27, 1-28.

Yu, J. (2009) 'Qualitative research on the attitudes toward teenage sexual behavior of Chinese British families: Methodological issues'. Journal of Transcultural Nursing, 20 (2), 156-63. 